\title{
ESTUDO DO EFEITO DA COLOSTOMIA PROXIMAL TERMINAL NA CICATRIZAÇÃO DE ANASTOMOSES COLO-CÓLICAS EM RATOS ${ }^{1}$
}

\author{
Marcelo Betim Paes Leme ${ }^{2}$ \\ Delcio Matos $^{3}$ \\ Pedro Ricardo de Oliveira Fernandes ${ }^{4}$ \\ Nicolau Maués da Serra-Freire ${ }^{5}$
}

Leme MBP, Matos D, Fernandes PRO, Serra-Freire NM. Estudo do efeito da colostomia proximal terminal na cicatrização de anstomoses colo-cólicas em ratos. Acta Cir Bras [serial online] 2002 Nov-Dez;17(6). Disponível em URL: http://www.scielo.br/acb.

RESUMO - Objetivo: Estudar os efeitos da colostomia proximal terminal na cicatrização de anastomoses colo-cólicas em ratos. Métodos: 36 ratas foram divididas em 2 grupos: grupo controle (C) com 12 animais submetidos à ressecção cólica segmentar seguida de anastomose colo-cólica primária, e grupo colostomizado $(\mathrm{CZ})$ com 24 animais submetidos ao mesmo procedimento do grupo $\mathrm{C}$ complementado com uma colostomia proximal. A cicatrização anastomótica foi avaliada em dois períodos distintos, $2^{\circ} \mathrm{e} 7^{\circ}$ dias de pós-operatório $(\mathrm{PO})$, em relação à deiscência anastomótica, aderências, epitelização mucosa, pressão de ruptura e variáveis histológicas. Os resultados foram submetidos a estudo estatístico considerando-se como significante valores de $p<0,05$. Resultados: A deiscência, principal variável analisada nessa pesquisa, não ocorreu em ambos os grupos estudados. As aderências foram significantemente mais intensas no grupo $\mathrm{C}$ no $7^{\circ} \mathrm{PO}$. Nos dois grupos, a ruptura intestinal sempre ocorreu ao nível da anastomose no $2^{\circ} \mathrm{PO}$; no $7^{\circ} \mathrm{PO}$, a maior parte das rupturas aconteceram na alça cólica fora da zona anastomótica (100\% do grupo C e $70 \%$ do grupo $\mathrm{CZ}$ ). A análise das demais variáveis demonstrou equivalência entre os dois grupos. Conclusão: Os resultados dessa pesquisa não demonstraram diferença significante entre anastomoses colo-cólicas em ratos associados ou não à colostomia proximal.

DESCRITORES - Colostomia. Cólon.

\section{INTRODUÇÃO}

A colostomia proximal tem sido considerada historicamente como um procedimento que confere maior segurança às suturas e anastomoses colo-cólicas e colo-retais, especialmente quando se trata de anastomoses de risco ${ }^{1,2,3}$.
Entretanto, essa possível proteção exercida pela colostomia vem sendo questionada por alguns autores 4, 5. Alguns trabalhos experimentais também têm demonstrado que a colostomia proximal não diminui a incidência de deiscência anastomótica ${ }^{6,7}$.

Tem-se considerado também como fator desfavorável ao uso das colostomias as taxas significativas

1. Trabalho realizado no Departamento de Cirurgia do Curso de Medicina do Centro Universitário de Volta Redonda/RJ - UniFOA.

2. Professor Assistente da Disciplina de Clínica Cirúrgica do Curso de Medicina do UniFOA e Mestre em Gastroenterologia Cirúrgica pela UNIFESP - EPM.

3. Professor Livre Docente e Chefe do Grupo de Coloproctologia da Disciplina de Gastroenterologia Cirúrgica da Escola Paulista de Medicina - UNIFESP.

4. Acadêmico Sextanista do Curso de Medicina do UniFOA e Presidente da Liga Acadêmica de Cirurgia Geral e do Trauma do UniFOA.

5. Professor Titular Livre Docente da Disciplina de Higiene e Saúde Pública e da Disciplina de Bioestatística do Curso de Medicina do UniFOA. 
de morbidade e mortalidade atribuídas a este procedimento $5,8,9,10$.

Outros autores, no entanto, relatam que, embora a freqüência de deiscência anastomótica não esteja diminuída quando protegidas pela colostomia, as complicações sépticas decorrentes da deiscência são melhores controladas na presença da colostomia ${ }^{11,12}$.

Assim, a inexistência de consenso sobre o papel da colostomia na proteção das anastomoses colocólicas, especialmente em colo não preparado, nos motivou à realização desta pesquisa que teve como objetivo avaliar o efeito da colostomia proximal na cicatrização de anastomoses colo-cólicas esquerdas em ratos.

\section{MÉTODOS}

Este trabalho foi elaborado e desenvolvido no Laboratório de Cirurgia Experimental do Curso de Medicina do Centro Universitário de Volta Redonda UniFOA e no Curso de Pós-Graduação da Disciplina de Gastroenterologia Cirúrgica da Escola Paulista de Medicina - UNIFESP.

Utilizou-se nesse estudo 36 ratas (Rattus Norvegicus Albinus) com idade de aproximadamente 120 dias e peso médio de $241 \mathrm{~g}$, que foram divididas aleatoriamente em 2 grupos: grupo controle (C) com 12 animais submetidos à ressecção cólica esquerda de $1 \mathrm{~cm}$, seguida de anastomose colo-cólica primária localizada $2 \mathrm{~cm}$ acima da reflexão peritonial, e grupo colostomizado (CZ) com 24 animais foi submetido ao mesmo procedimento do grupo C complementado com uma colostomia proximal-terminal.

Os ratos receberam anestesia inalatória com éter sulfúrico e o acesso à cavidade peritonial foi realizado através de uma celiotomia mediana de $5 \mathrm{~cm}$ de extensão.

As anastomoses foram realizadas em plano único de sutura seromuscular (extramucoso) com 8 a 10 pontos separados, confeccionados com fio de polipropileno 6-0 com agulha cilíndrica. Utilizou-se uma lupa cirúrgica com aumento de $4 \mathrm{X}$ para melhor visualização do procedimento operatório.

A colostomia proximal-terminal foi realizada no grupo $\mathrm{CZ}$, pela secção do colo na flexura cólica esquerda e exteriorização da boca proximal no quadrante superior esquerdo do abdome. A alça distal foi suturada com pontos contínuos e deixada livre na cavidade peritonial.

Os ratos foram sacrificados com dose inalatória letal de éter no $2^{\circ} \mathrm{e} 7^{\circ} \mathrm{PO}$, para estudo das anastomoses em relação à deiscência anastomótica, aderências, epitelização mucosa, pressão de ruptura e variáveis histológicas.

Considerou-se deiscência anastomótica, a presença de abscesso perianastomótico, peritonite difusa ou da própria deiscência. As aderências foram classificadas como ausentes, ou se presentes, de acordo com o número de quadrantes que ocupavam.

Após a avaliação macroscópica da cavidade, um segmento de $4 \mathrm{~cm}$ do colo esquerdo, contendo a anastomose centralizada, foi ressecado para a avaliação da sua resistência e verificação da epitelização da ferida mucosa. Para a realização do teste de resistência a peça cirúrgica foi imersa em cuba contendo água e o ar insuflando de $10 \mathrm{em} 10 \mathrm{mmHg}$, a cada 15 segundos, até que se percebesse o borbulhar de ar na água evidenciando a ruptura da peça ${ }^{13,14}$. As pressões foram registradas em um manômetro, graduado em $\mathrm{mmHg}$, conectado em Y ao sistema. Posteriormente, a peça foi aberta pelo bordo antimesenterial, através de uma incisão longitudinal. A área interna da anastomose foi inspecionada e avaliada a epitelização mucosa da zona anastomótica, que foi classificada considerando-se o número de terços epitelizados na linha anastomótica.

Dois fragmentos da zona anastomótica, um correspondente à borda mesenterial e outro à anti-mesenterial da alça cólica, foram submetidos ao estudo histológico com coloração pela hematoxilina-eosina. Na região cicatricial, foram estudados pela microscopia óptica quanto a sua presença e intensidade, as variáveis histológicas: necrose, inflamação aguda, inflamação crônica, regeneração mucosa, fibrose e tecido de granulação. $\mathrm{O}$ seguinte critério foi estabelecido para a quantificação das alterações: $0=$ ausência de alteração, $1=$ grau leve de alterações, 2 = grau moderado de alterações, $3=$ grau intenso de alterações. $O$ examinador das lâminas histológicas não estava informado a respeito da origem dos espécimes operatórios, se de anastomoses do grupo $\mathrm{C}$ ou $\mathrm{CZ}$.

Os resultados foram analisados estatisticamente pelo teste " $z$ " para comparação entre proporções, teste " $\boldsymbol{t}$ " para comparação entre médias e teste "Qui Quadrado de Pearson" para comparação entre proporções independentes, considerando-se como significante valores de $\mathrm{p}<0,05^{15,16}$.

\section{RESULTADOS}

Durante o experimento foram registrados 3 óbitos, todos em decorrência de complicações da anestesia. A deiscência anastomótica, principal variável analisada neste trabalho, não ocorreu nos dois grupos estudados tanto no $2^{\circ}$ quanto no $7^{\circ}$ dia. Verificou-se também que não ocorreram complicações da colostomia. 
As aderências perianastomóticas estiveram presentes em ambos os grupos nos dois períodos analisados. No $7^{\circ}$ dia observou-se maior freqüência de aderências perianastomóticas intensas, que ocupavam 3 ou 4 quadrantes, no grupo C (100\%) que no grupo $\mathrm{CZ}(70 \%)$. Essa diferença foi estatisticamente significante $(\mathrm{p}<0,05)$.

Em relação aos níveis de pressão de ruptura não foi possível se detectar diferença entre os grupos nos dois períodos estudados, $2^{\circ}$ e $7^{\circ}$ dia (Tabela 1). Quando se comparou as pressões registradas no $2^{\circ}$ dia com as pressões registradas no $7^{\circ}$ dia, nos 2 grupos, verificouse que as pressões no $2^{\circ}$ dia foram sistematicamente mais baixas que no $7^{\circ}$ dia. Essa diferença foi estatisticamente significante $(\mathrm{p}<0,05)$.

TABELA 1 - Pressão média de ruptura em mmHg.

\begin{tabular}{c|c|c|c}
\hline Sacrifício & Grupo C & Grupo CZ & $\mathrm{p}$ \\
\hline $2^{\circ} \mathrm{PO}$ & 106,00 & 94,17 & $\mathrm{p}>0,05$ \\
$7^{\circ} \mathrm{PO}$ & 248,33 & 223,50 & $\mathrm{p}>0,05$ \\
\hline
\end{tabular}

Teste " $z$ ".

Pode-se verificar ainda que, no $2^{\circ}$ dia, as rupturas ocorreram com maior freqüência na anastomose e que, no $7^{\circ}$ dia, ocorreram com maior freqüência na alça intestinal (Gráfico 1). No grupo $\mathrm{C}$, no $7^{\circ} \mathrm{PO}$, todas rupturas ocorreram na parede intestinal, contudo, no grupo $\mathrm{CZ}, 30 \%$ das rupturas ocorreram na anastomose.

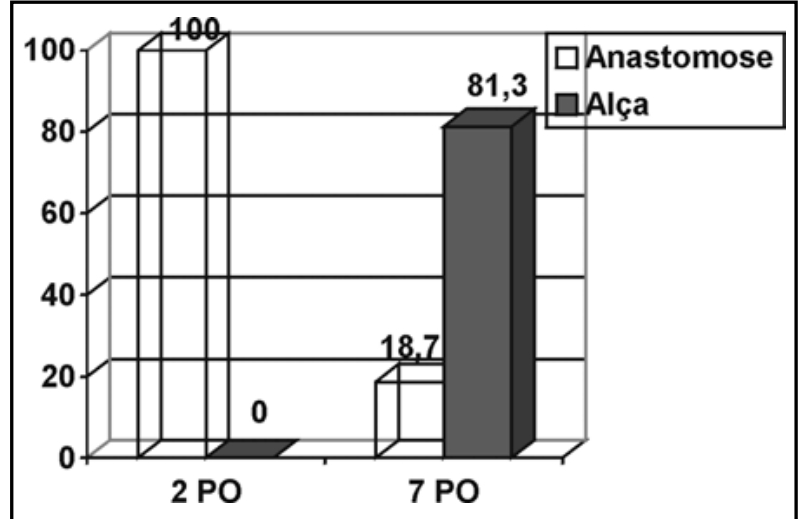

GRÁFICO 1 - Frequência do local de ruptura em percentual comparação entre o $2^{\circ}$ e $7^{\circ} \mathrm{PO}$. Qui-quadrado p<0,05.

Em relação a epitelização mucosa observou-se que a freqüência de cobertura epitelial $>2 / 3$ na linha anastomótica, foi menor no grupo C (20\%) que no grupo $\mathrm{CZ}(83,3 \%)$ no $2^{\circ}$ dia de pós-operatório (PO.). Essa diferença foi significativa $(\mathrm{p}<0,05)$.

O estudo histológico da zona anastomótica revelou equivalência, na maioria das variáveis analisadas, entre os dois grupos estudados, tanto no $2^{\circ}$ quanto no $7^{\circ}$ PO.

\section{DISCUSSÃO}

A deiscência anastomótica tem sido considerada a principal complicação das operações do intestino grosso. Alguns procedimentos têm sido utilizados na tentativa de diminuir a freqüência da deiscência anastomótica nas operações colo-retais, entre eles, a colostomia proximal. Existem, entretanto, controvérsias sobre o papel "protetor" das colostomias na cicatrização das anastomoses.

Experimentalmente, BLOMQUIST et al. ${ }^{17} \mathrm{e}$ UDÉN et al. ${ }^{18}$ descreveram uma maior incidência de deiscência anastomótica na ausência de colostomia proximal. SENAGORE et al. ${ }^{19}$ verificaram não haver diferenças significativas entre o grupo com e sem colostomia proximal, entretanto BIELECKI et al. ${ }^{6}$ observaram uma maior taxa de deiscência anastomótica comprovada radiologicamente no grupo com colostomia proximal. Nesse trabalho, a deiscência anastomótica foi a principal variável analisada, todavia, este evento não ocorreu em ambos os grupos nos dois períodos estudados.

As aderências estiveram presentes nos dois grupos estudados, entretanto foram significantemente mais intensas no grupo $\mathrm{C}$ no $7^{\circ} \mathrm{PO}$. Talvez, a colostomia proximal-terminal, ao impedir o trânsito fecal na porção cólica anastomosada, diminua a intensidade de reação inflamatória na zona anastomótica, um dos fatores responsáveis pelo estímulo à formação de aderências. BIONDO-SIMÕES ${ }^{13}$ e AGUILAR-NASCIMENTO et al. ${ }^{20}$ também demonstraram uma grande freqüência de aderências perianastomóticas após anastomoses cólicas.

O teste de resistência das anastomoses demonstrou que a pressão média de ruptura foi equivalente nos 2 grupos tanto no $2^{\circ}$ quanto no $7^{\circ} \mathrm{PO}$. Entretanto, verificamos que, no $2^{\circ} \mathrm{PO}$, a pressão de ruptura foi significantemente menor que no $7^{\circ} \mathrm{PO}$ em ambos os grupos. Isto possivelmente se explica pelo fato de que a resistência da anastomose, na fase inicial da cicatrização, é mais dependente das suturas e, nas fases mais tardias, a reparação tecidual e a deposição de colágeno estão definitivamente mais presentes ${ }^{14,21,22,23}$. 
Nesse experimento, verificamos ainda que, no $7^{\circ}$ $\mathrm{PO}$, as rupturas ocorreram com maior freqüência fora da zona anastomótica. Tem-se verificado na literatura que, na segunda fase da cicatrização, a região anastomótica torna-se mais resistente à ruptura que a parede da alça intestinal ${ }^{21,24,25}$.

A incidência de $30 \%$ de rupturas na anastomose no grupo $\mathrm{CZ}$, no $7^{\circ} \mathrm{PO}$, pode ser atribuída à possibilidade da colostomia proximal interferir na resistência da anastomose, como também verificado por BLOMQUIST et al. ${ }^{17}$ e UÉDEN et al. ${ }^{18}$. Entretanto, outras pesquisas não verificaram alterações significativas na resistência das anastomoses submetidas à colostomia proximal ${ }^{6,19}$.

A verificação de melhor epitelização mucosa na região anastomótica no $2^{\circ} \mathrm{PO}$, no grupo $\mathrm{CZ}$, talvez possa ser explicada pela ausência de trânsito e contato fecal na zona anastomótica, o que teoricamente poderia minimizar as agressões à linha anastomótica.

O estudo dos eventos histológicos entre os dois grupos revelou equivalência na maioria dos itens estudados. KORUDA e ROLANDELLI ${ }^{26} \mathrm{e}$ HENDRIKS e MASTBOOM ${ }^{23}$ relataram que esta semelhança nos achados histológicos se deve principalmente à seqüência natural de eventos cicatriciais que ocorrem de forma muito semelhante no processo de regeneração tecidual de qualquer anastomose intestinal.

\section{CONCLUSÃO}

Os resultados verificados nesse experimento não mostraram diferença significante entre a cicatrização de anastomoses colo-cólicas em ratos associadas ou não à colostomia proximal terminal.

\section{REFERÊNCIAS}

1. McSherry CK, Grafe WR, Perry HS, Glenn F. Surgery of the large bowel for emergent conditions. Arch Surg 1969, 98:749-53.

2. Goligher JC, Graham NG, Dombal FT. Anastomotic dehiscence after anterior resection of rectum and sigmoid. Br J Surg 1970, 57:109-18.

3. Valerio D, Jones PF. Immediate resection in treatment of large bowel emergencies. Br J Surg 1978, 65:712-6.

4. Kiss DS, Bocchini SF. Anastomose em plano único de sutura na cirurgia cólica de urgência. Rev Paul Med 1976, 87:112-4.

5. Wara P, Sorensen K, Berg V. Proximal fecal diversion: review of ten years experience. Dis Colon Rectum 1981, 24:114-9.

6. Bielecki K, Grotowski M, Kalczac M. nfluence of proximal end diverting colostomy on the healing of left side colonic anastomosis: an experimental study in rats. Int $\mathrm{J}$ Colorectal Dis 1995, 10:193-6.

7. Paes Leme MB, Matos D, Luderer LA, Fonseca DA. Avaliação do efeito da colostomia proximal na cicatrização de anastomoses colo-cólicas em ratos com obstrução intestinal. Rev Col Bras Cir 2001, 28(2):109-15.

8. Hopkins JE. Transverse colostomy in the management of cancer of the colon. Dis Colon Rectum 1971, 14:232-6.

9. Porter JA, Salvati EP, Rubin RJ, Eisenstat TE. Complications of colostomies. Dis Colon Rectum 1989, 32:299-303.

10. Winkler MJ, Volpe PA. Loop transverse colostomy: the case against. Dis Colon Rectum 1982, 25:321-6.

11. Karanjia ND, Corder AP, Holdsworth PJ, Heald RJ. Risk of peritonitis and fatal septicaemia and the need to defunction the low anastomosis. Br J Surg 1991, 78:196-8.

12. Pakkastie TE, Ovaska JT, Pekkala ES, Luukkonen PE, Jarvinen HJ. A randomized study of colostomies in low colorectal anastomosis. Eur J Surg 1997, 163:929-33.

13. Biondo-Simões MLP. Estudo comparativo da evolução de anastomoses do cólon esquerdo realizadas na vigência ou na ausência de obstrução aguda, com e sem limpeza, no rato [Tese - Doutorado]. Escola Paulista de Medicina - UNIFESP; 1994.

14. Cronin K, Jakson DS, Dumphy JE. Changing bursting strength and collagen content of the healing colon. Surg Gynecol Obstet $1968,126: 747-53$

15. Sounis E. Bioestatística: princípios fundamentais, metodologia estatística, aplicação às ciências biológicas. 1ed. Rio de Janeiro: McGraw-Hill do Brasil; 1971.

16. Guedes MLS, Guedes JS. Bioestatística para profissionais de saúde. 1ed. Brasília: CNPq; 1988.

17. Blomquist $\mathrm{P}$, Jiborn $\mathrm{H}$, Zederfeldt B. Effect of diverting colostomy on breaking strength of anastomosis after resection of left side of the colon. Am J Surg 1985, 149:712-5.

18. Udén P, Blomquist $\mathrm{P}$, Jiborn $\mathrm{H}$, Zederfeldt B. Influence of proximal colostomy on the healing of left colon anastomosis: an experimental study in rat. Br J Surg 1988, 75:325-9.

19. Senagore A, Milsom JW, Walshaw RK, Dunstan R, Chaudry IH. Does a proximal colostomy affect colorectal anastomotic healing ? Dis Colon Rectum 1992, 35:182-5.

20. Aguilar-Nascimento JE, Caporossi C, Figueiredo PC, Alves DC, Kobata CM, Chacon JP. Anastomose primária em plano único na obstrução aguda do cólon esquerdo: estudo em ratos. Rev Bras Colo-Proct 1990, 10:193-6.

21. Herrmann JB, Woodward SC, Pulaski EJ. Healing of colonic anastomosis in the rat. Surg Gynecol Obstet 1964, 119:269-75.

22. Ballantyne GH. Intestinal suturing: review of the experimental foundations for tradicional doctrines. Dis Colon Rectum 1983, 26:836-43

23. Hendriks T, Mastboom WB. Healing of experimental intestinal anastomosis: parameters for repair. Dis Colon Rectum 1990, 33:891-901.

24. Jiborn H, Ahonen J, Zederfeldt B. Healing of experimental colonic anastomosis: bursting strength of the colon after left colon resection and anastomosis. Am J Surg 1978, 136:587-94.

25. Aguilar-Nascimento JE, Mathie RT, Man WK. Enhanced intraanastomotic healing by operative lavage with nutrient solutions in experimental left-sided colonic obstruction. Br J Surg 1995, $82: 461-4$

26. Koruda MJ, Rolandelli RH. Experimental studies on the healing of colonic anastomosis. J Surg Res 1990, 40:504-15.

27. Segreti EM, Levenback C, Morris M, Lucas CR, Gershenson DM, Burke TH. A comparasion of end loop colostomy for fecal diversion in gynecologic patients with colonic fistulas. Gynecol Oncol 1996, 60:49-53. 
Leme MBP, Matos D, Fernandes PRO, Serra-Freire NM. Effect of proximal terminal colostomy on the healing of colonic anastomosis in rats. Acta Cir Bras [serial online] 2002 Nov-Dec;17(6). Available from URL: http://www.scielo.br/acb.

ABSTRACT - Objective: To evaluate the effects of the proximal-terminal colostomy on the healing of colonic anastomosis in rats. Methods: 36 rats were allocated into two groups: control group (C) with 12 rats subjected to colonic ressectinon and primary anastomosis; colostomy group $(\mathrm{CZ})$ with 24 rats subjected to the same procedure done in the group $\mathrm{C}$, but complemented with a proximal-terminal colostomy. Anastomosis healing was evaluated at the $2^{\text {nd }}$ and $7^{\text {th }}$ postoperative days (PO) by the presence of anastomotic dehiscence and mucosal line, adhesions, bursting pressure, and histologic examination. Results: There was no anastomotic dehiscence in the two groups. Adhesions were more intense in group $\mathrm{C}$ at the $7^{\text {th }} \mathrm{PO}$. At both groups, the intestinal rupture was always on the anastomotic line at the $2^{\text {nd }} \mathrm{PO}$; at the $7^{\text {th }} \mathrm{PO}$, most of ruptures happened on the colonic zone out of the anastomotic line (100\% of group C and $70 \%$ of group $\mathrm{CZ}$ ). No significant difference was noticied in the other healing anastomotic variables. Conclusion: There is no difference in the healing of colonic anastomosis related to the presence or abscence of a proximal colostomy in rats.

KEY WORDS - Colostomy. Colon.

Conflito de interesse: nenhum

Fonte de financiamento: nenhuma

Endereço para correspondência:

Prof. Dr. Delcio Matos

R. Edison, 278/61

04618-031 São Paulo - SP

dmatos.dcir@epm.br

Data do recebimento: 03/09/2002

Data da revisão: $21 / 09 / 2002$

Data da aprovação: 08/10/2002 